\title{
The Interaction Between Intra-Cyclic Variation of the Velocity and Mean Swimming Velocity in Young Competitive Swimmers
}

Authors

Affiliations
T. M. Barbosa ${ }^{1}$, P. G. F. Morouço ${ }^{2}$, S. Jesus ${ }^{3}$, W. G. Feitosa ${ }^{4}$, M. J. Costa ${ }^{5}$, D. A. Marinho ${ }^{6}$, A. J. Silva ${ }^{7}$, N. D.Garrido

Affiliation addresses are listed at the end of the article
Key words

swimming

- kinematics

- swim strokes

- speed fluctuation

age-group swimmers accepted after revision

March 27, 2012

\section{Bibliography}

Dol http://dx.doi.org/

10.1055/s-0032-1312582

Published online: 2012

Int J Sports Med

(c) Georg Thieme

Verlag KG Stuttgart · New York

ISSN 0172-4622

\section{Correspondence}

Prof. Tiago Miguel Barbosa

IPB

Sport Sciences

Campus Sta Apolonia

5300 Bragança

Portugal

Tel.: +35/127/3330664

Fax: $+35 / 127 / 3313684$

barbosa@ipb.pt

\section{Abstract}

$\nabla$

The aim of this study was to assess the relationship between the intra-cyclic variation of the horizontal velocity ( $\mathrm{dv}$ ) and the velocity of the 4 competitive swimming techniques in young swimmers. 45 young swimmers performed a set of maximal $4 \times 25 \mathrm{~m}$ (freestyle, backstroke, breaststroke and butterfly stroke) swims with in water start. A speed-meter cable was attached to the swimmer's hip. The dv and the swimming velocity were analyzed. Within-subject tests presented significant variations in the $\mathrm{dv}$ based on the swimming technique. Post-hoc test

\section{Introduction}

\section{$\nabla$}

The aim of a competitive swimmer is to travel the event distance as fast as possible:

$\sum_{\mathrm{i}=1}^{\mathrm{n}} t_{i}=\frac{\sum_{i=1}^{n} d_{i}}{\sum_{i=1}^{n} v_{i}}$

Where $t_{\mathrm{i}}$ is the duration of each partial phase of the swimming event (start, turns, swimming strokes/laps and finish), $d_{\mathrm{i}}$ is the swimmer's centre of mass displacement during each partial moment and $v_{\mathrm{i}}$ is swimmer's centre of mass velocity during each phase. Experimental research has been conducted regarding the importance of each partial phase for swimming performance [e.g. [18]. In several of those studies it was verified that the swimming moment is the most important to predict the performance [e.g. [18]. In this sense, the best swimming performance predictor is the mean clean velocity and/or mid-pool swimming velocity. Theoretically it can be considered [9]:

$v_{\max }=\frac{\dot{E}_{\text {tot-max }}}{C}$ revealed significant differences across all pairwised swimming techniques $(\mathrm{P}<0.001)$, except for the comparison between freestyle and backstroke $(\mathrm{P}=0.98)$. The dv was higher in the breaststroke, followed by the butterfly, the backstroke and the freestyle. The quadratic models had the best goodness-of-fit and the lower error of estimation for the relationship between the $\mathrm{dv}$ and the swimming velocity in all swimming techniques $\left(0.24 \leq R^{2} \leq 0.51\right)$. As a conclusion, there is a non-linear relationship where the increase of swimming velocity leads to a decrease of $\mathrm{dv}$ in young competitive swimmers.

Where $v_{\max }$ is the maximal swimming velocity, $\dot{\mathrm{E}}_{\text {tot-max }}$ is the maximal total energy expenditure corrected for body mass and $C$ is the energy cost (a ratio between energy expenditure and distance). Scientific evidence has been collected about these relationships in the 4 swimming techniques [7]. Since the mid-seventies there has been some speculation that the $C$ would be related to the intra-cyclic variations of the horizontal velocity (dv) of the body. In classic mechanics energy is defined as the capacity to produce mechanical work. A theoretical comparison between the mechanical work performed while swimming at constant $v$ and with $\mathrm{d} v$ is described as [25]:

$$
\begin{aligned}
\frac{w_{d}}{w_{d-\text { constan }}}= & 1+\frac{3}{T} \int_{0}^{T}\left[\frac{\Delta v(t)}{v_{0}}\right] d t+\frac{3}{T} \int_{0}^{T}\left[\frac{\Delta v(t)}{v_{0}}\right]^{2} d t \\
& +\frac{1}{T} \int_{0}^{T}\left[\frac{\Delta v(t)}{v_{0}}\right]^{3} d t
\end{aligned}
$$

where $w_{\mathrm{d}}$ is the mechanical work, $v(t)$ is the swimming velocity at a given time, $v_{0}$ is the swimming velocity at the beginning of the stroke 
cycle and $T$ is the total duration of the stroke cycle. Changes in $v$ of $\sim 10 \%$, within a stroke cycle, results in an additional work demand of $\sim 3 \%$ [25]. However, probably due to the difficulty to assess mechanical work in aquatic locomotion techniques, it seems there is no experimental data confirming this. Instead of mechanical work, it is easier to estimate energy cost, which is a variable related to it. It was suggested that a higher $d v$ leads to an increase in $C$ to overcome inertia and drag force. Later on experimental data confirmed these assumptions in the 4 swimming strokes $[4,5]$.

Relating eqs. 2 and 3 it can be speculated that there is some kind of relationship between $v$ and $d v$. At least for land-based locomotion techniques, the relationship is described as being nonlinear [22]. There is a self-paced $v$ at which the energy expenditure is the lowest [12]. Added to that, some data reports a positive relationship between vertical displacement of the hip and $C$ or mechanical work or efficiency for land-based locomotion techniques $[12,19]$ and between $d v$ and $C$ for aquatic-based locomotion techniques $[4,5,34]$. So, the $d v$ can be considered as an estimator of the energy cost of locomotion [34]. Conversely, experimental evidences about the $d v-v$ relationship for competitive swimming techniques are not consensual. Literature reports that: (i) there is no significant $d v-v$ relationship assessing the hip with a speed-meter $[28,29]$ or the 3D kinematics of the centre of mass [27] in front crawl; (ii) decreases of the $d v$ are associated with linear increases of $v$ for the hip with a speed-meter in breaststroke [21], a 2D kinematics in butterfly stroke [31] or a 3D kinematics in breaststroke [30]; (iii) there is a quadratic relationship assessing the 2D kinematics of the centre of mass in all 4 swim strokes [5]. So, there is a good chance to clarify this issue as no solid evidence is reported in the literature about the $d v-v$ relationship.

This is the first study to analyze all 4 strokes for a single group of swimmers in order to clearly verify which kind of $d v-v$ relationship exists. Training sessions for swimmers aged 12-14 years should be mainly based on technique drills to improve all 4 swimming techniques. Unfortunately, most of the time the swimmers in this age group are submitted to very heavy conditional training and are not very concerned with technique. On the one hand swimmers in this age group should already have a fairly good and consolidated technique. Young swimmers seems to be the appropriate cohort group to compare the 4 swimming techniques since they practice all of them on a daily basis. On the other hand, adult/elite swimmers are at a stage of high-specialization in their competitive career, mostly concentrating on one single swimming technique and sometimes on a specific swimming event (i.e., a single swimming technique and distance). Therefore, the intra-subject comparison and the assessment of the relationship of selected kinematical variables in all 4 swimming strokes in adult/elite swimmers seems to have major limitations, which can be avoided when assessing young swimmers. Additionally, to the best of our knowledge, scientific evidence about the $d v-v$ relationship in young swimmers is non-existent. The aim of this study was to assess the $d v-v$ relationship of the 4 competitive swimming techniques in young swimmers. Based on the data reported in the literature for land-based locomotion techniques, it was hypothesized that for swimming techniques there is also a non-linear $d v-v$ relationship.

\section{Materials and Methods}

$\nabla$

\section{Subjects}

45 young swimmers ( 23 boys and 22 girls) with at least 4 years of experience in competitive swimming, participating on a regular basis in regional and national level competitions at the moment of data collection, volunteered as subjects $(12.78 \pm 1.15$ years-old, $1.59 \pm 0.10 \mathrm{~m}$ of height, $49.34 \pm 10.15 \mathrm{~kg}$ of body mass, $2.57 \pm 0.43$ Tanner stages by self-evaluation). Parents and coaches gave their written consent for the swimmers' participation in this study. All procedures were in accordance with the Declaration of Helsinki and approved by the Review Board of the Institution. Additionally, this study has been performed in accordance with the ethical standards proposed by Harriss and Atkinson [17].

\section{Protocol}

The swimmers carried out a standard warm-up of approximate $1500 \mathrm{~m}$ including continuous swim at low-moderate intensity, drills for legs and arms and some sprints at the end. Each swimmer undertook a set of maximal $4 \times 25 \mathrm{~m}$ (freestyle, backstroke, breaststroke and butterfly stroke) swims with an underwater start in a randomized order. Participants performed the bout alone with no other swimmer in the lane or nearby lanes to reduce drafting and pacing effects, or being affected by extra drag force due to exogenous factors. The swimmers were advised to reduce gliding during the start to allow data collection between the $11^{\text {th }}$ and $24^{\text {th }} \mathrm{m}$. Passive recovery of at least $30 \mathrm{~min}$ took place between the warm-up and the first bout, as well as, between each of the 4 bouts.

\section{Data collection}

A speed-meter cable (Swim speedo-meter, Swimsportec, Hildesheim, Germany) was attached to the swimmer's hip. The speed-meter was placed in the forehead-wall of the swimming pool, about $0.2 \mathrm{~m}$ above water surface. Young swimmers' coaches and researchers are aware that weak kicking is a major issue in young swimmers. Although it seems there is no solid scientific evidence on that, it was assumed that the absolute contributions of kicking to total velocity might be even lower in children than in adult swimmers ( 10-15\%). Therefore, the turbulent action of the water or kicking the cable and inducing data collection errors might be almost negligible for these young swimmers. During data collection an evaluator made a visual inspection of the curves being drawn in the software's interface while the swimmer performed the bout. If some technical/methodological issue happened (e.g., bumping feet on the cable), evaluators asked the swimmers to repeat the bout once again.

Bio-signal was acquired on-line at a sampling rate of $50 \mathrm{~Hz}$. A LabVIEW® (v. 2009) software interface was used to acquire, display and process pair-wise velocity-time data on-line during the swim bouts. To transfer data from the speed-meter to the software application, a 12-bit resolution acquisition card (USB6008, National Instruments, Austin, Texas, USA) was used as well. The integrated system (speed-meter plus software) was extensively described and validated for the $d v$ with Doppler effect [10] and videometric system [11]. The same data collection technique (e.g. $[14,15,23,35]$ ) and even the commercial apparatus, specially the hardware, had already been used and reported in several other papers (e.g. [20,28]).

Data was exported to a signal processing software (AcqKnowledge v.3.5, Biopac Systems, Santa Barbara, USA) and filtered with a $5 \mathrm{~Hz}$ cut-off low-pass $4^{\text {th }}$ order Butterworth. The selection of 
the cut-off value was done according to residual analysis (residual error vs. cut-off frequency). The hip's $d v$ was analyzed as $[4,5,34]$ :

$$
d v=\frac{\sqrt{\frac{\sum_{i}\left(v_{i}-v\right)^{-2} \cdot F_{i}}{n}}}{\frac{\sum_{i} v_{i} \cdot F_{i}}{n}} \cdot 100
$$

where $d v$ represents the intra-cyclic variation of the horizontal velocity of the hip, $v$ represents the mean swimming velocity, $v_{\mathrm{i}}$ represents the instant swimming velocity, $F_{\mathrm{i}}$ represents the acquisition frequency and $n$ is the number of observations. For further analysis the $d v$ mean value of 3 consecutive stroke cycles between the $11^{\text {th }} \mathrm{m}$ and $24^{\text {th }} \mathrm{m}$ from the starting wall was considered.

The hip's $d v$ it is not exactly the same as the centre of mass $d v[3,16,26]$. In spite of the same $v$-time graph profile, there are 2 slight bias: (i) a temporal delay between major peaks in the anatomical landmark and the centre of mass of approximately $0.1 \mathrm{~s}$ (i.e., $\sim 10 \%$ of the stroke cycle duration); (ii) the $v$ range is slightly higher for the hip than for the centre of mass in approximately $0.2 \mathrm{~m} / \mathrm{s}$ in front crawl and backstroke (i.e., $~ 10 \%$ of the maximal velocity) and $0.4 \mathrm{~m} / \mathrm{s}$ in butterfly and breaststroke (i.e., $\sim 15 \%$ of the maximal velocity). The videometric system has some limitations, such as: (i) expensive apparatus to collect and process data; (ii) some errors due to data collection and bio-signal processing and; (iii) methodological procedures are very time consuming. Those limitations are even higher when collecting data in an aquatic environment than in a terrestrial one. On the other hand, the speed-meter is more "coach-friendly" since it is an affordable piece of equipment, easy to operate allowing almost instant feed-back for swimmers. Considering the strong and weak points of each technique it seems that the speed-meter, although there is some slight bias, is an appropriate procedure to assess the swimmer's kinematics. This is important when a large number of participants is assessed, reducing the time spend in data collection and data processing, which is relevant whenever convenience samples are selected, as in this research. Nevertheless, using the fixed anatomical landmark as a measure, the associated errors should always be taken into consideration.

\section{Statistical procedures}

Normality was determined by the Shapiro-Wilk test. The homoscedasticity assumption was checked with the Levene test. Box plots with quartile data from $d v$ and $v$ were calculated for each swimming technique, including mean values of 3 consecutive stroke cycles per swimmer. Mean $\pm 1 \mathrm{SD} d v$ curves normalized to the stroke cycle duration were computed with MATLAB ${ }^{\circledR}$ (v.7.11.0, MathWorks Inc., Massachusetts, USA) for each swimming technique.

Repeated measures (within-subjects ANOVA) analysis of the $d v$ according to the swimming technique was performed. The repeated measures (within-subjects ANCOVA) interaction between the $d v$ and the $\Delta v$ according to the swimming technique $(\mathrm{P} \leq 0.05)$ was assessed. For the ANCOVA, the $\Delta v$ was defined as being the swimming velocity amplitude (maximal velocity minus minimal velocity in the 4 bouts). ANOVA and ANCOVA repeated measures were followed by Bonferroni tests.
The level of statistical significance was set at $\mathrm{P} \leq 0.05$. Effect size was computed based on Eta-squared $\left(\mathrm{n}^{2}\right)$ procedure, and values interpreted as being: without effect if $0<\eta^{2} \leq 0.04$; minimum if $0.04<\eta^{2} \leq 0.25$; moderate if $0.25<\eta^{2} \leq 0.64$ and; strong if $\eta^{2}>0.64$.

Linear and polynomial regressions ( $2^{\text {nd }}$ and $3^{\text {rd }}$ power) between $d v$ and $v$ were computed and compared for each swimming technique. For each case the mathematical model that presented the best goodness-of-fit and the lowest standard error of the estimation was adopted. For all cases, polynomial regressions in $2^{\text {nd }}$ power were the selected ones. Reported were the fit line equations, the coefficients of determination $\left(R^{2}\right)$, the adjusted coefficients of determination $\left(R_{a}^{2}\right)$ and the standard errors of the estimation (s). The level of statistical significance was set at $\mathrm{P} \leq 0.05$. As a rule of thumb, it was considered a: small effect size if $0 \leq|\mathrm{r}| \leq 0.2$; (ii) moderate effect size if $0.2<|\mathrm{r}| \leq 0.5$ and; (iii) strong effect size if $|\mathrm{r}|>0.5$.

\section{Results}

\section{$\nabla$}

For qualitative assessment, $\bullet$ Fig. 1 presents the mean curves of $d v$ normalized to the stroke cycle. In freestyle and backstroke $d v$ was characterized by a multi-peak profile. In breaststroke and butterfly stroke $d v$ was characterized by 2-peak and 3-peak profiles, respectively.

For quantitative assessment, $\odot$ Fig. 2 presents the box plot of the $d v$ ( $\odot$ Fig. 2, panel A) and the $v(\diamond$ Fig. 2, panel B). Within-subject test (ANOVA) presented meaningful variations in the $d v$ based on the swimming technique $[\mathrm{F}(3,132)=718.968 ; \mathrm{P}<0.001$; $\left.\mathrm{n}^{2}=0.92\right]$. Post-hoc test revealed significant differences across all pair-wised swimming techniques $(\mathrm{P}<0.001)$, except for the comparison between freestyle and backstroke $(\mathrm{P}=0.98)$. The $d v$ was higher in the breaststroke, followed by the butterfly, the backstroke and the freestyle. Conversely, within-subject test with the $\Delta v$ as covariate (ANCOVA) presented non-significant interaction in the $d v$ with a moderate effect size $[\mathrm{F}(3,129)=1.691$; $\left.\mathrm{P}=0.95 ; \mathrm{\eta}^{2}=0.26\right]$.

- Table 1 presents the fit line equation for the $d v-v$ relationship. The quadratic models were the ones with the best goodness-of-fit and the lower error of estimation for all swimming techniques. All mathematical models were statistically significant with a moderate-strong effect size $\left(0.24 \leq R^{2} \leq 0.51 ; 0.48 \leq r \leq 0.71\right)$. 0 Fig. 3 presents, for each one of the 4 swimming techniques, the $d v-v$ relationship plus fit line and $95 \%$ confidence interval.

\section{Discussion}

$\nabla$

The aim of this study was to assess the $d v-v$ relationship in the 4 competitive swimming techniques in young swimmers. The $d v$ was higher in the breaststroke, thereafter in the butterfly, the backstroke and the freestyle, respectively. Polynomial regressions in $2^{\text {nd }}$ power were the ones with the best goodness-of-fit and the lower error of estimation for the $d v-v$ relationships in all swim techniques. There are 2 main points to be selected for the $d v$ 's assessment: (i) one fixed anatomical landmark, notably the hip, or; (ii) the swimmer's overall centre of mass. Centre of mass kinematics are supposed to be much more valid than hip's kinematics (e.g., $[3,16,26])$. The hip's velocity peak presents a slight time-delay ( 10\% stroke cycle total duration) and some bias in the instantaneous velocity 

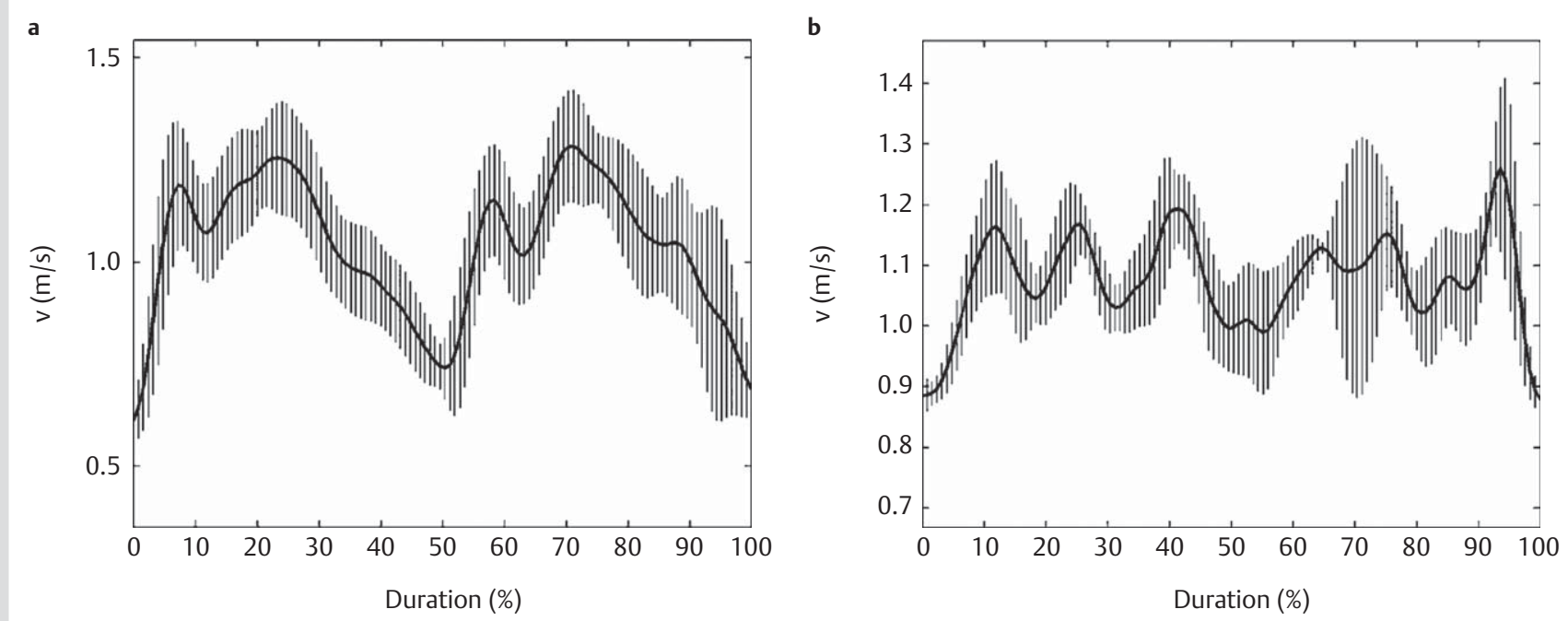

c

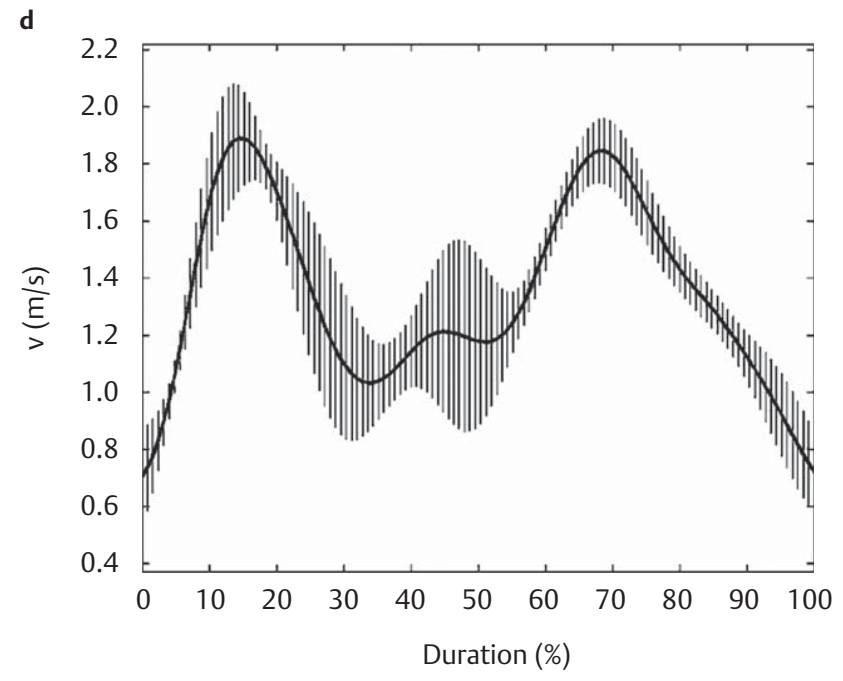

Fig. 1 Mean \pm 1SD of the intra-cyclic variation of the horizontal velocity normalized to the stroke cycle duration in front crawl (panel a), backstroke (panel b), breaststroke (panel c) and butterfly stroke (panel d).

and $d v$ in comparison to the centre of mass kinematics (e.g., $[3,16,26])$.

The fixed point assessment can be made with mechanical techniques (e.g., cable or propeller based speed-meters and accelerometers), image-based techniques (e.g., image digitalization) and mixed ones (e.g. intermittent light-trace photography); while nowadays the centre of mass is mainly assessed with image-based techniques [34]. Within all the procedures available, the image-based techniques are often considered as the gold standard. Nevertheless, some weakness are apparent in the image-based techniques [34]: (i) digitalization process is very time-consuming: (ii) anthropometric biomechanical model used to compute the inter-limb inertial effects must be valid and accurate; (iii) it ignores the additional inertial effect of the added mass of water; (iv) it imposes several anatomical landmark reference points to be digitized that are limited by images quality (e.g., distortions, water bubbles and waves, parallax errors); (v) the $2 \mathrm{D}$ or 3D kinematics assessment imply different and complex procedures (e.g., hidden points, number of cameras, space references, calibration, coordinates and algorithm for 3D reconstruction); (vi) reduced interactivity with coaches and swimmers to deliver information quickly. Some weaknesses of the mechanical apparatus should be considered: (i) it only assesses an anatomical landmark; (ii) the mechanical connection to the subject might impose data bias (i.e., its consistency and the incapability to monitor the effective inertia of the swimmer's body). On the other hand, main strengths of the mechanical velocimetry include: (i) it is an affordable technique; (ii) less time consuming, allowing data collection for larger samples; (iii) it highly interactive, delivering quickly useful data to swimmers and coaches. So, considering a balance between strengths and weaknesses of each method, the subjects' characteristics and on the top of that the moderately large sample size adopted, the hip's kinematics assessed with mechanical velocimetry seems to be a good solution.

Swimming is an aquatic locomotion technique based on periodic arms, legs and trunk actions leading to changes in the velocity within a stroke cycle:

$v=v_{0}+\Delta v(t)$

where $v$ is the swimmer's mean velocity, $v_{0}$ is the swimmer's velocity at the beginning of the stroke cycle, $\Delta v$ is the variation of the swimming velocity throughout the stroke cycle and $t$ is 
the time. The $d v$, considering a given period of time, defines the swimmer's acceleration and it is dependent upon the applied resultant force and the inertial term of the second Newton equation:

$F=m \cdot a$

Where $F$ is the resultant force, $m$ is the body mass and $a$ is the acceleration. In competitive swimming the horizontal resultant force is the balance between propulsion and drag forces. The inertial term includes the swimmer's body mass plus and sometimes the added water mass. The body's acceleration represents the $d v$. So, eq. 6 is developed as [34]:

$\operatorname{Pr}+D=(B M+m) \cdot a$

Where $P r$ is the total propulsive forces, $D$ is the drag force, $B M$ is the swimmer's body mass, $m_{\mathrm{a}}$ is the added water mass and $a$ is
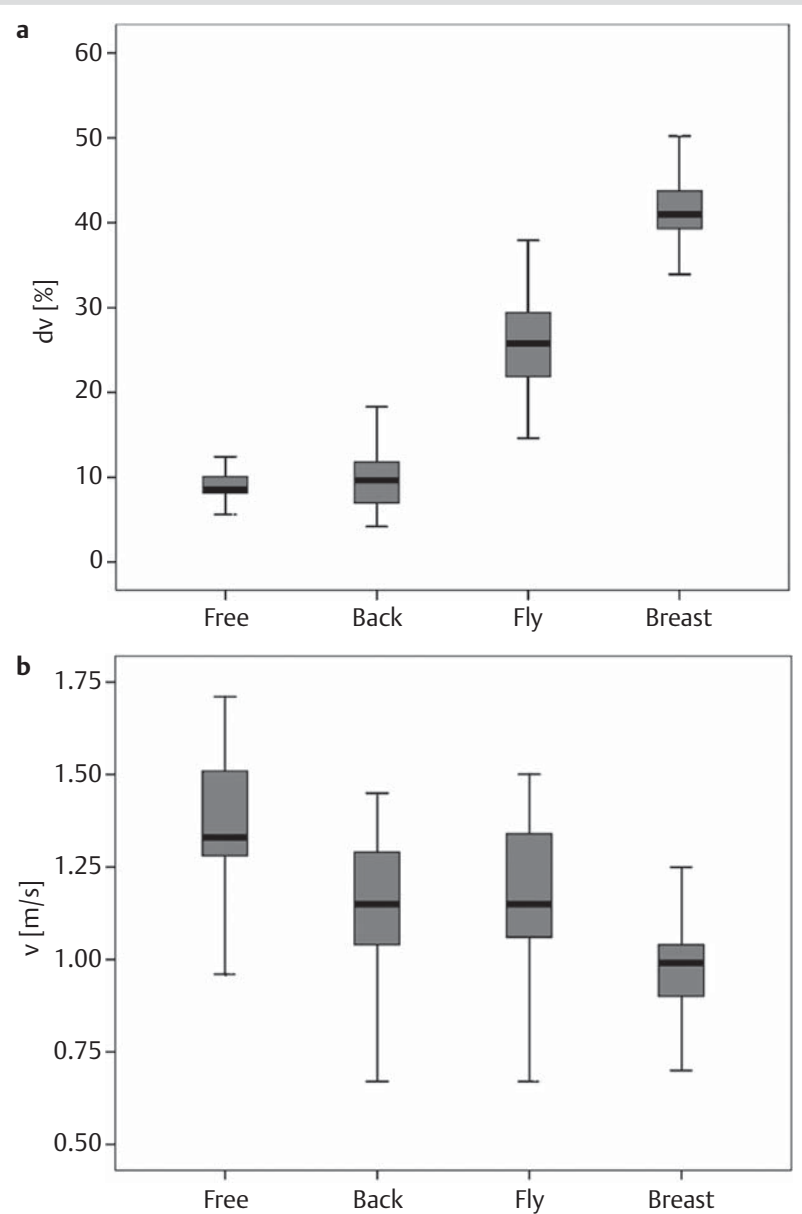

Fig. 2 Quartile distribution of the intra-cyclic variation of the horizontal velocity (panel a), and swimming velocity (panel b) for the 4 swimming techniques. the swimmer's acceleration (i.e., $\mathrm{a}=d v$ ). Re-arranging now eq. 7 towards the swimmer's acceleration (i.e., $d v$ ):

$d v=\frac{P r+D}{B M+m_{a}}$

So, from a theoretical point of view, the $d v$ represents a balance between propulsive and resistive forces, being an efficiency estimator. Gaining both theoretical and experimental data about this topic is one of the most challenging and interesting projects in this scientific field [34]. On a regular basis the energy cost it is reported as being related to mechanical, propulsive and overall efficiency [9]. So, the energy cost it is an estimator of the movement efficiency, where an increase of the first means a decrease of the latter. Due to the complexity of assessing the efficiency directly, researchers sometimes decided to estimate it through the energy cost of swimming. Not only theoretical research, as reported previously, but also experimental research demonstrated that a higher energy cost (i.e., lower efficiency) is related to a higher $d v$ in all swimming strokes. High variations on $d v$ also impose a high energy cost, since extra energy must be delivered to overcome inertial forces [34]. Based on this, the $d v$ is considered as an informative kinematic variable to analyze the overall swimmers mechanics because it allows the [9]: (i) identification of critical issues within the stroke cycle; (ii) collection of relevant data for practitioners and; (iii) the categorisation of the swimmer's competitive level.

At freestyle and backstroke $d v$ was characterized by a multipeak profile. These multi-peak profiles were similar to what was described for both techniques assessing a fixed point [15] and the centre of mass [8]. 2 major peaks/curves were related to each arm's actions and several small peaks due to the changes of propulsive phases throughout the arm's underwater path. The kicking action in front crawl and backstroke provides propulsion, as well as stabilization. These propulsive kick phases would likely show as several small peaks in velocity profile trace. So, the small peaks might not just be from the arm action throughout the underwater stroke, but also from the leg kick action.

In breaststroke and butterfly strokes, $d v$ was characterized by a 2-peak and a 3-peak profile, respectively. At Breaststroke, one major peak is related to the arm's action and the other one to the leg's actions $[8,15]$. In the transition between arm's and leg's actions, the standard deviation increases. This can be related to another peak that some swimmers might do, as described elsewhere [21]. The variation in limb's actions seems to be related to: (i) the added mass inertial effect during gliding [13] or, (ii) a lack of inter-limb coordination that imposes a significant velocity decrease [20]. In Butterfly stroke, one major peak is related to the first kick during arm's entry $[2,3,23]$. The second peak is related to a more latero-medial component of the hand's path, promoting an increased contribution from the insweep to propulsion [2,3]. The third peak might be related to the upsweep movement of the arms combined with the second kick, before the arm's exit $[3,22]$. Often in young swimmers, one of the 2 kicks per stroke cycle is not performed (either as the hands enter

$\begin{array}{llllll} & \text { Equation } & \mathbf{R}^{2} & \mathbf{R}_{\mathbf{a}}{ }^{2} & \mathbf{P} & \mathbf{S} \\ \text { free } & \mathrm{d} v=-37.30 \cdot v-12.127 \cdot \mathrm{v}^{2}+36.923 & 0.35 & 0.32 & <0.001 & 2.267 \\ \text { back } & \mathrm{d} v=-19.062 \cdot \mathrm{v}+4.088 \cdot \mathrm{v}^{2}+25.758 & 0.43 & 0.43 & <0.001 & 2.339 \\ \text { breast } & \mathrm{d} v=-72.576 \cdot \mathrm{v}+31.922 \cdot \mathrm{v}^{2}+80.653 & 0.24 & 0.17 & 0.03 & 3.141 \\ \text { fly } & \mathrm{d} v=-34.941 \cdot \mathrm{v}-7.765 \cdot \mathrm{v}^{2}+55.607 & 0.51 & 0.49 & <0.001 & 4.011\end{array}$

Table 1 Fit line equations for the interplay between the intra-cyclic variation of the horizontal velocity (dv) and the swimming velocity (v) in each swimming technique. 

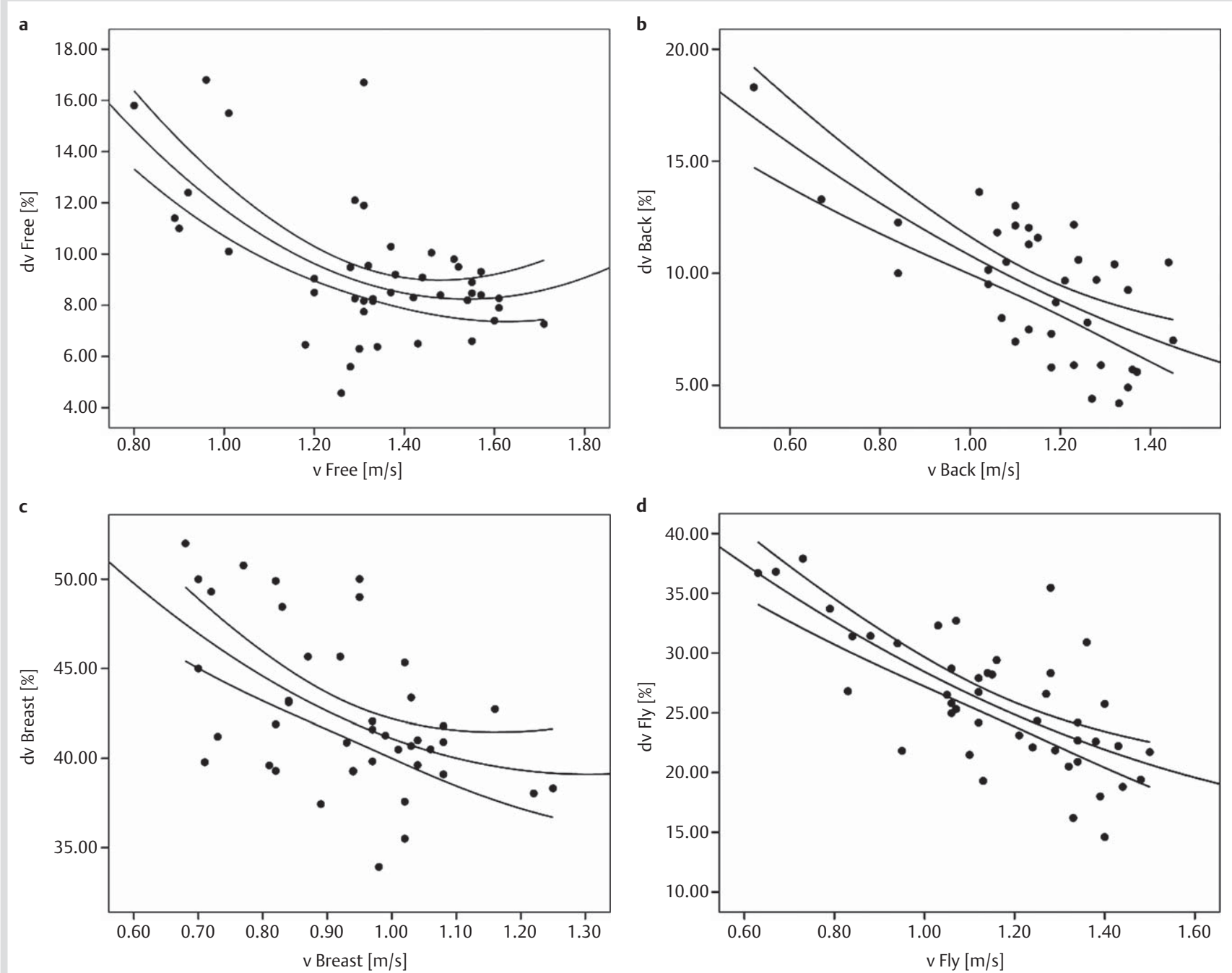

Fig. 3 Scattergram plus fit line and $95 \%$ confidence interval of the relationship between the intra-cyclic variation of the horizontal velocity (dv) and the swimming velocity ( $v$ ) in front crawl (panel a), backstroke (panel b), breaststroke (panel c) and butterfly stroke (panel d).

or as they exit the water). All participants in this research were instructed in the training sessions to perform 2 kicks per stroke cycle on a daily basis. This seems to happen as the major peak occurs at the beginning of the stroke cycle (arm's entry plus first kick) and at the end of it (upsweep and arm's exit plus second kick).

There were significant $d v$ differences across almost all pairwised swimming techniques. The $d v$ presented the highest value in the breaststroke, then in the butterfly, in the backstroke and finally in the freestyle, respectively. Similar data was presented with adult/elite swimmers [14]. However, in this case, an intersubject comparison was performed where the backstroke presented a lower $d v$ than the freestyle. Since all the swimmers did not perform the 4 swimming techniques during data collection, probably there were differences in the competitive level of each cohort group leading to these results. It seems there is a pathflow between the swimmer's energetics, kinematical and kinetic profile. The swimmer's energetics depend on his/her kinematic behaviour and this depends on the kinetics [9]. Some papers demonstrated that when comparing energetics, kinematics and kinetics of the 4 swimming techniques all of these follow more or less the same pattern. The energy expenditure [6], the tethered swimming force [24] and the intra-cyclic mechanical impulse $[1,2,32,33]$ are higher in breaststroke, thereafter in butterfly, backstroke and freestyle in this order. These data suggest that actually there might be relationships among the swimmers' energetics, kinematics and kinetics profiles. Probably, swimming techniques producing higher forces (at least when tethered) impose a higher intra-cyclic mechanical impulse, $d v$ and energy expenditure. Some partial relationships between these variables were already reported, e.g., the energy expenditure versus $d v$ relationship [4,5]. However, to the best of our knowledge a research relating to all of them in a single research paper has not been published up to now.

The quadratic models were the ones with the best goodness-offit and the lowest error of estimation for all swimming techniques. As for land-based locomotion techniques, the $d v-v$ relationship is described as being non-linear [22]. Walkers and runners have a self-selected velocity in which there is a lower $d v$ and probably a lower energy cost [12]. Although in land-based locomotion techniques there is some discussion between 2 main theories explaining gait (inverted pendulum vs. 6 determinants of gait) both of them aim to understand how walkers increase their movement efficiency. Inverted pendulum theory is related to conservation of the mechanical energy [19]. It suggests that to cost less energy, stance leg has to act like an inverted pendulum 
and describe such an arc. Conversely, the 6 determinants of gait theory [19] propose that the pelvis rotation, the pelvis oscillation, the knee flexion at the support phase, the foot mechanics, the knee mechanics and the lateral displacing of the pelvis can reduce the displacement of the centre of mass and therefore improve gait efficiency [19]. It is acceptable for 2 theories to differ, but they serve the same goal of reducing the energy cost in an opposing rather than complementary fashion. Although gait and swimming are not the same kind of locomotion actions, it seems that the 6 determinants theory is not only valid for gait but also for $d v$ in swimming. Both theories are based on the premise that vertical and horizontal centre of mass displacements/velocity cost energy.

In all 4 swimming techniques, the increases of the $v$ lead to a decrease of the $d v$. This type of $d v-v$ relationship was already reported once for elite swimmers in a $2 \mathrm{D}$ centre of mass kinematics study [5], although another study did not verify this in a 3D centre of mass kinematics study [27]. In the paper by Barbosa et al. [5] an inter-subject instead of a within-subject assessment was carried out. Probably Psycharakis et al. [27] did not find any relationship because an assessment was made in a much lower range of velocities than in this research and that of Barbosa et al. [5]. This might suggest that $d v$ it is not sensitive to very small changes in the $v$ as happens from lap to lap throughout a short distance event such as the 200-m freestyle. On the other hand, Barbosa et al. [5] established such a relationship in a wider range of swimming velocities. Swimmers made a set of 7 bouts ranging from low to maximal velocities, which increased the range of velocities assessed.

Coaches training young swimmers should spend a large part of the training sessions presenting technique drills and giving feed-backs about the swimmer's technique instead of focusing on heavy physical conditioning. According to swimming periodization programs and career plans, the main goal of training programs for young swimmers (aged between approximately 12-14 years) must be to improve their technical ability in all 4 swimming techniques. The main trend of this research was that swimmers able to achieve a higher $v$, seem to have a lower $d v$. Thus, a training session should be focused on technical drills to improve technique (i.e., increase swimming efficiency and reduce $d v$ at maximal pace).

In summary, in the 4 swimming techniques, there is a non-linear $d v-v$ relationship. Swimmers, as walkers and runners, have a non-linear relationship where the increase of the velocity leads to a decrease of the $d v$ and probably a lower energy cost, since these last 2 variables are also linked.

\section{Affiliations}

${ }^{1}$ IPB, Sport Sciences, Bragança, Portugal

${ }^{2}$ Research Center for Human Movement Sciences, Polytechnic Institute of Leiria, Leiria, Portugal

${ }^{3}$ Sport Sciences, Polytechnic Institute of Bragança/CIDESD, Bragança, Portugal

${ }^{4}$ Sport Sciences, University of Ceará, Fortaleza, Brazil

${ }^{5}$ Departement of Sport Sciences, Polytechnic Institute of Bragança, Bragança, Portugal

${ }^{6}$ Sport Sciences, UBI, Covilhã, Portugal

${ }^{7}$ Exercise and Health Sciences Department, University of Trás-os-Montes and Alto Douro, ports, Vila Real, Portugal

\section{References}

1 Alves F. Average resultant impulse per phase in swimming: a tool for technical analysis. In: Abrantes J (ed). Proceedings of the XIV International Symposium on Biomechanics in Sports. Lisbon: Faculty of Human Movement, 1996; 281-284

2 Barbosa T, Santos Silva J, Sousa F, Vilas-Boas JP. Measurement of butterfly average resultant impulse per phase. In: Ginaikellis K (ed). Proceedings of the XXth International Symposium on Biomechanics in Sports. Cáceres: University of Extremadura, 2002; 35-38

3 Barbosa T, Santos Silva JV, Sousa F, Vilas-Boas JP. Comparative study of the response of kinematical variables from the hip and the center of mass in butterfliers. In: Chatard JC (ed). Biomechanics and Medicine in Swimming IX. St Etienne: University of St Etienne Publications, 2003; 93-98

4 Barbosa TM, Keskinen KL, Fernandes RJ, Colaço PC, Lima AB, Vilas-Boas $J P$. Energy cost and intra-cyclic variations of the velocity of the centre of mass in butterfly stroke. Eur J Appl Physiol 2005; 93: 519-523

5 Barbosa TM, Lima F, Portela A, Novais D, Machado L, Colaço P, Gonçalves $P$, Fernandes $R$, Keskinen K, Vilas-Boas JP. Relationships between energy cost, swimming velocity and speed fluctuation in competitive swimming strokes. In Vilas- Boas JP, Alves F, Marques A (eds.). Biomechanics and Medicine in Swimming X Porto: Port J Sports Sci. 2006; a 192-194

6 Barbosa TM, Fernandes RJ, Keskinen KL, Colaço PC, Cardoso C, Silva J, Vilas-Boas JP. Evaluation of the energy expenditure in competitive swimming strokes. Int J Sports Med 2006; 27: 894-899

7 Barbosa TM, Fernandes RJ, Keskinen KL, Vilas-Boas JP. The influence of stroke mechanics into energy cost of elite swimmers. Eur J Appl Physiol 2008; 103: 139-149

8 Barbosa TM, Silva AJ, Reis AM, Costa MJ, Garrido ND, Policarpo FB, Reis VM. Kinematical constrictions swimming front crawl and breaststroke with the Aquatrainer ${ }^{\circledR}$ snorkel. Eur J Appl Physiol 2010; 109: 1155-1162

9 Barbosa TM, Bragada JA, Reis VM, Marinho DA, Carvalho C, Silva AJ. Energetics and biomechanics as determining factors of swimming performance: updating the state of the art. J Sci Med Sports 2010; b 13: 262-269

10 Barbosa TM, Costa MJ, Morais JE, Jesus S, Marques MC, Batista J, Gonçalves J. Conception, development and validation of a software interface to assess human's horizontal intra-cyclic velocity with a mechanical speedo-meter. In: Siny Jan S, Feipel V, Aerenhout D, Baeyens JP, Carpentier A, Cattrysse E, Clarys JP, Duchateau J, Guissard N, Leloup T, Provyn S, Rooze M, Scafoglieri A, Schuind F, Roy PV, Warzée N (eds.). Proceedings of the XXIIIrd Congress of the International Society of Biomechanics. Brussels: Université Libre de Brussels \& Université D’Europe. Brussels: 2011 a; 217

11 Barbosa TM, Costa MJ, Morais JE, Jesus S, Silva AJ, Batista J, Gonçalves J. Validation of an integrated system to assess horizontal intra-cyclic velocity with a mechanical speedo-meter. In: Vilas-Boas JP, Machado L, Kim W, Veloso AP (eds.). Biomechanics in Sports 29. Porto: Port J Sports Sci, 2011; b 833-835

12 Cavanagh $P, \operatorname{Kram} R$. The efficiency of human movement - a statement of the problem. Med Sci Sports Exerc 1985; 17: 304-308

13 Colman V, Persyn U, Ungerechts B. A mass of water added to the swimmer's mass to estimate the velocity in dolphin-like swimming below the water surface. In: Keskinen KL, Komi PV, Hollander AP (eds). Biomechanics and Medicine in Swimming VIII. Jyvaskyla: Gummerus Printing, 1999; 89-94

14 Craig $A B$, Pendergast $D R$. Relationships of stroke rate, distance per stroke, and velocity in competitive swimming. Med Sci Sports Exerc 1979; 11: 278-283

15 Craig AB, Termin B, Pendergast DR. Simultaneous recordings of velocity and video during swimming. In: Vilas- Boas JP, Alves F, Marques A (eds.). Biomechanics and Medicine in Swimming X. Porto: Port J Sports Sci, 2006; 32-35

16 Figueiredo P, Vilas-Boas JP, Maia J, Gonçalves P, Fernandes RJ. Does the hip reflect the centre of mass swimming kinematics? Int J Sports Med 2009; 30: 779-781

17 Harriss DJ, Atkinson G. Update - ethical standards in sport and exercise science research. Int J Sports Med 2011; 32: 819-821

18 Hay J, Guimarães A. A quantitative look at swimming biomechanics. Swim Techn 1983; 20: 11-17

19 Кио A. The six determinants of gait and the inverted pendulum analogy: a dynamic walking perspective. Hum Mov Sci 2007; 26: 617-656

20 Leblanc H, Seifert L, Tourny-Chollet C, Chollet D. Intra-cyclic distance per stroke phase, velocity fluctuation and acceleration time ratio of a breaststroker's hip: a comparison between elite and non-elite swimmers at different race paces. Int J Sports Med 2007; 28: 140-147 
21 Manley $P$, Atha J. Intra-stroke velocity fluctuations in paces breaststroke swimming. In: MacLaren D, Reilly T, Lees A (eds.). Biomechanics and Medicine in Swimming VI. London: E \& FN Spon, 1992; 151-160

22 Minetti A. The three modes of terrestrial locomotion. In: Nigg B, MacIntosh B, Mester J (eds.). Biomechanics and Biology of Movement. Illinois: Human Kinetics, 2000; 67-78

23 Morouço $P$, Lima AB, Semblano $P$, Fernandes $D$, Gonçalves $P$, Sousa $F$, Fernandes RJ, Barbosa TM, Correia MV, Vilas-Boas JP. Validation of a cable speedometer for butterfly evaluation. In: Vilas- Boas JP, Alves F, Marques A (eds.). Biomechanics and Medicine in Swimming X. Porto: Port J Sports Sci, 2006; 236-238

24 Morouço P, Keskinen KL, Vilas-Boas JP, Fernandes RJ. Relationship between tethered forces and the four swimming techniques performance. J Appl Biomech 2011; 27: 161-169

25 Nigg B. Selected methodology in biomechanics with respect to swimming. In: Hollander AP, Huijing PA, de Groot G9 (eds.). Biomechanics and Medicine in Swimming IV. Champaign, Illinois: Human Kinetics, 1983; 72-80

26 Psycharakis SG, Sanders RH. Validity of the use of a fixed point for intracycle velocity calculations in swimming. J Sci Med Sports 2009; 12: 262-265

27 Psycharakis S, Naemi R, Connaboy C, McCabe C, Sanders RH. Threedimensional analysis of intracycle velocity fluctuations in front-crawl swimming. Scand J Med Sci Sports 2010; 20: 128-135

28 Schnitzler C, Seifert L, Ernwein V, Chollet D. Arm coordination adaptations assessment in swimming. Int J Sports Med 2008; 29: 480-486

29 Seifert L, Toussaint H, Schnitzler C, Alberty M, Chavallard F, Lemaitre F, Vantorre J, Chollet D. Effect of velocity increase on arm coordination, active drag and intra-cyclic velocity variations in front crawl. In: Nomura T, Ungerechts B (eds.). $1^{\text {st }}$ International Scientific Conference of Aquatic Space Activities. Tsukuba: University of Tsukuba, 2008; 254-259
30 Takagi H, Sugimoto S, Nishijima N, Wilson B. Differences in stroke phases, arm-leg coordination and velocity fluctuation due to event, gender and performance level in breaststroke. Sports Biomech 2004; 3: $15-27$

31 Togashi T, Nomura T. A biomechanical analysis of the swimmer using the butterfly stroke. In: MacLaren D, Reilly T, Lees A (eds.). Biomechanics and Medicine in Swimming VI. London: E \& FN Spon, 1992; 87-91

32 van Tilborgh L, Willems E, Persyn $U$. Estimation of breaststroke propulsion and resistance-resultant impulses from film analyses. In: Ungerechts B, Wilke K, Reischle K (eds.). Swimming Science V. Illinois: Human Kinetics Books, 1988; 67-71

33 Vilas-Boas JP, Santos P. Comparison of swimming economy in three breaststroke techniques. In: Miyashita M, Mutoh Y, Richardson A (eds.). Medicine and Science in Aquatic Sports. Bassel: Karger, 1994; 48-54

34 Vilas-Boas JP, Barbosa TM, Fernandes RJ. Speed fluctuation, swimming economy, performance and training in swimming. In: Seifert L, Chollet D, Mujika I (eds.). World Book of Swimming: From Science to Performance. New York: Nova Science Publishers, 2010; 119-134

35 Vilas-Boas JP, Costa L, Fernandes RJ, Ribeiro J, Figueiredo P, Marinho DA, Silva AJ, Rouboa A, Machado L. Determination of the drag coefficient during the first and second gliding positions of the breaststroke underwater stroke. J Appl Biomech 2010; 26: 324-331 